
approches théoriques et méthodologiques

\title{
Contribution de la classification à facettes pour l'organisation des connaissances dans les organisations
}

The Contribution of Faceted Classification to Knowledge Organization in

Enterprises

\section{Orélie Desfriches Doria}

\section{OpenEdition}

Journals

Édition électronique

URL : http://journals.openedition.org/edc/3889

DOl : 10.4000/edc.3889

ISSN : 2101-0366

Éditeur

Université Lille-3

\section{Édition imprimée}

Date de publication : 1 décembre 2012

Pagination : 173-200

ISBN : 978-2-917562-08-6

ISSN : $1270-6841$

\section{Référence électronique}

Orélie Desfriches Doria, « Contribution de la classification à facettes pour l'organisation des connaissances dans les organisations », Études de communication [En ligne], 39 | 2012, mis en ligne le 01 décembre 2014, consulté le 01 mai 2019. URL : http://journals.openedition.org/edc/3889 ; DOI

10.4000/edc.3889

Ce document a été généré automatiquement le 1 mai 2019.

(c) Tous droits réservés 


\title{
Contribution de la classification à facettes pour l'organisation des connaissances dans les organisations
}

\author{
The Contribution of Faceted Classification to Knowledge Organization in \\ Enterprises
}

Orélie Desfriches Doria

\section{NOTE DE L'ÉDITEUR}

Cette recherche a bénéficié du soutien de l'ANR Miipa-Doc n² 2008 CORD 01403

\section{Introduction}

1 L'organisation des connaissances est un champ large qui concerne de nombreuses communautés: informatique, Sciences de l'information et de la documentation, bibliothèques, gestion des connaissances (Knowledge Management), sociologie des sciences, et au-delà. Si l'on considère les espaces où se concentrent les problématiques de l'organisation des connaissances, on en distingue trois principaux, le Web, les bibliothèques, et les organisations de type entreprises. Les objectifs, les processus et les principes d'organisation des connaissances dans ces trois types d'espaces diffèrent à plusieurs niveaux: quantité d'information, dispositifs de recherche d'information traditionnellement disponibles, types d'utilisateurs, activités mises en œuvre, finalité des espaces évoqués.

2 L'organisation des connaissances fait écho à des expressions variées dans la littérature, comme la gestion des connaissances, l'ingénierie des connaissances, l'organisation du 
savoir, la capitalisation des connaissances, les systèmes d'organisation des connaissances, le partage des savoirs, le management des connaissances, la mémoire organisationnelle. Hjørland distingue deux sens principaux pour cette expression. Dans son sens restreint, l'organisation des connaissances évoque des activités comme la description de documents, l'indexation et la classification, exercées dans les bibliothèques, les bases de données bibliographiques, les archives [...] par les bibliothécaires, les archivistes, les spécialistes de l'information, les spécialistes de certains sujets, ainsi que par les algorithmes informatiques et les profanes. Dans son sens plus large, Hjørland considère que l'organisation des connaissances a à voir avec l'organisation sociale du travail intellectuel comme l'organisation des universités et autres institutions de recherche et d'éducation, la structure des disciplines et des métiers, l'organisation sociale des médias, la production et la dissémination du savoir (Hjørland, 2008).

Pour Cotte, la définition «qui recouvrirait le plus la notion d'organisation des connaissances au sens strict est que les organisations se préoccupent de recenser, qualifier, classifier, organiser, partager le volume global de connaissances que les salariés produisent, brassent, manipulent » (2007, p. 69).

4 Les Systèmes d'Organisation des Connaissances (SOC) jouent ici un rôle fondamental d'articulation entre les utilisateurs, les interfaces, les éventuels indexeurs et les connaissances contenues dans les supports, en normalisant le vocabulaire employé pour la description et la recherche des ressources. Dans cet article, nous nous intéresserons à un SOC spécifique, la classification à facettes, au service de l'organisation des connaissances dans les entreprises. D'après Mills (2004), le processus cognitif qui connecte les producteurs de textes stockés et les destinataires imaginés du savoir peut être efficacement assisté par la classification à facettes.

Dans le cadre du projet ANR Miipa-Doc, nous travaillons sur une application proposant une classification à facettes adaptative basée sur le tagging de documents de toutes natures, et une méthodologie de construction progressive de cette classification par les utilisateurs eux-mêmes, dans laquelle certaines dimensions de description peuvent être partagées par un groupe métier, projet, ou au sein de l'ensemble de l'entreprise.

Dans une première partie nous rappellerons les besoins des organisations en matière d'organisation des connaissances, puis nous focaliserons sur l'approche traditionnelle de la classification à facettes. Dans une troisième partie nous présenterons les apports d'une approche de la classification à facettes adaptée à l'organisation des connaissances dans l'entreprise, en présentant l'outil sur lequel nous travaillons.

\section{Besoins spécifiques des organisations en matière d'organisation des connaissances}

7 Il est communément admis que les connaissances représentent un capital pour les organisations. Par conséquent, leur valorisation peut générer des profits par l'amélioration de la rentabilité du travail (Cotte, 2008). Pour Teulier et Girard, qui voient l'organisation comme «le produit de l'activité des acteurs, le savoir-être ensemble pour produire quelque chose » (2005, p. 393), les connaissances cruciales pour les entreprises sont les connaissances pour l'action. Ces dernières sont considérées comme telles lorsque l'on peut rendre compte de «leur mise en œuvre, leur concrétisation dans une action pour atteindre un but $»(2005$, p. 392). 
Une entreprise dispose généralement d'un organigramme qui permet aux acteurs de situer les services et les personnes dans la hiérarchie. Cela ne permet pas pour autant au centre de décision de l'entreprise de disposer de toutes les connaissances disponibles dans l'entreprise. Plus précisément, celles-ci résident dans les cerveaux de tous les acteurs de l'entreprise, dans les documents stockés sur divers supports, dont les postes informatiques individuels, et dans leur créativité et leur expertise, soutenues par ces documents, pour inventer des stratégies afin de répondre aux objectifs fixés par la hiérarchie. Dans cette perspective, les documents constituent donc un support à l'expertise des acteurs et nous pensons que leur contextualisation améliore les capacités d'adapter le savoir qu'ils possèdent déjà.

par Les connaissances formalisées, celles qui sont inscrites dans des documents, apparaissent donc, malgré leur volume, comme une partie seulement de l'ensemble des connaissances produites dans l'exercice d'un métier. Des méthodologies existent pour faire émerger les connaissances tacites comme celle proposée par Nonaka et Takeushi (1997), mais cette partie formalisée des connaissances d'une organisation et consignée dans des documents reste fragile. En effet, la mondialisation des marchés, la libéralisation de l'économie et l'impact des Technologies de l'Information et de la Communication (TIC) accélèrent les processus de décision, en même temps que l'entreprise évolue dans un espace à trois dimensions (Grundstein, 2002) : une dimension globale, une dimension locale et une dimension d'influence caractérisée par des interactions avec d'autres organisations. Cela rend nécessaire les supports informatisés car ils sont dé-corrélés de la présence physique ; les connaissances sont donc disséminées sur de multiples postes et autres supports dont la pérennité est toujours à surveiller. D'autre part, ces mêmes facteurs engendrent une instabilité dans l'environnement des entreprises, qui aboutit à des besoins de contextualisation des informations. Ainsi la forme d'incertitude que génère cette situation se répercute sur les $\mathrm{SOC}$ qui décrivent une réalité évolutive et sujette à interprétation (Boydens, 2010). Cet état de fait nécessite selon nous un assouplissement des principes des $\mathrm{SOC}$ utilisés dans les entreprises afin d'adapter les outils de gestion de l'information à cet environnement en mouvement constant.

Dans les années 2000, la littérature issue de l'ingénierie des connaissances se concentre sur des approches formelles, qui tendent par exemple à aboutir à des objectifs de construction d'une mémoire organisationnelle définie comme "une représentation persistante, explicite, désincarnée des connaissances et des informations dans une organisation » (Gandon et Dieng-Kuntz, 2005, p. 142), ou encore à des approches basées sur des modélisations successives de l'organisation, des tâches et des agents (Tort et al., 2005), ou des méthodes comme MASK, basée elle aussi sur des modélisations (Ermine, 2000). Mais de nombreux auteurs se sont aussi intéressés à une approche contextualisée des connaissances (Argyris, 1995; Grundstein, 2003 ; Mahé, 2005; Sauvagnac et Falzon, 2000 ; Teulier et Girard, 2005) qui souligne l'importance de connaître le contexte de création des connaissances lors de leur réutilisation dans un nouveau contexte.

11 Par opposition aux bibliothèques, qui visent à organiser le savoir scientifique matérialisé dans des supports pérennes et dont le format est abouti, avec des SOC de nature plutôt formelle, en imaginant un utilisateur type, nous considérons qu'il est possible d'utiliser les mêmes types de SOC dans les entreprises (classifications, bases de données, thésaurus, index...) mais en assouplissant certains de leurs principes. Ainsi, les documents d'entreprise présentent certaines des caractéristiques proposées par Zacklad (2005) à propos des Documents Pour l'Action (DOPA) : l'inachèvement prolongé, la fragmentation, 
la circulation rapide du document, la pluri-auctorialité (plusieurs auteurs) et un rapport non trivial entre les parties $d u$ document. Ces caractéristiques des documents d'entreprise rendent, selon nous, nécessaire l'adaptation des SOC mis à disposition des acteurs dans l'entreprise, afin de pouvoir relater leur contexte de création.

L'outil que nous développons présente la possibilité d'assouplir certains principes de la classification à facettes dans ce but.

\section{Approche traditionnelle de la classification à facettes pour l'organisation des connaissances}

13 La problématique de l'organisation des connaissances est liée au savoir que les organisations veulent et tentent de conserver. Ces informations sont de natures diverses (données techniques, informations ou documents à valeur juridique, archives, données non structurées...) et elles sont consignées sur des supports variés (Internet, Intranets, postes de travail individuels, serveurs, disques durs).

\subsection{Documentarisation et Classification à facettes}

14 La documentarisation, qui consiste «à transcrire ou à enregistrer une production sémiotique sur un support pérenne puis à l'équiper d'attributs spécifiques visant à faciliter les pratiques liées à son exploitation ultérieure dans le cadre de la préservation de transactions communicationnelles distribuées» (Zacklad, 2005, p. 158), constitue un enjeu majeur dans la conservation et la transmission du savoir, en permettant l'accès aux documents et aux contenus.

15 La mémorisation des informations liées au processus de documentarisation, qui peuvent être d'ordre technique (contenu), organisationnel (coordination), de contexte (activité) ou de localisation (accès à un document), représente un effort conséquent que les $\mathrm{SOC}$ viennent soutenir (Pikas, 2007).

\subsection{Les classifications bibliographiques}

classifications bibliographiques sont généralement énumératives, organisées sous forme hiérarchique et basées uniquement sur la relation générique/spécifique, qui peut se traduire par «partie de » ou «sorte de » (Beghtol, 2001). Un schéma énumératif est caractérisé par le fait qu'il construit une vue d'ensemble de l'univers des pensées et des choses, dans une séquence continue avec une notation développée pour chaque terme, du plus générique au plus spécifique (Sayers, 1955 cité par Begthol, 2007). Les hiérarchies divisent et subdivisent les choses en groupes dans lesquels chaque nouveau groupe est une sous-espèce de son groupe parent. Ainsi, le principe d'héritage des propriétés se traduit par le fait que tout ce qui est vrai d'un groupe est aussi vrai de ses sous-groupes. Elles sont généralement dotées d'un système de notation.

17 Le problème principal de ces classifications est l'indexation par sujets (Mills, 2004), et la difficulté à représenter des sujets composés ou pouvant appartenir à plusieurs branches d'une arborescence ou dépendants de plusieurs disciplines. Les principes de la classification à facettes sont élaborés spécifiquement pour pouvoir décrire des sujets complexes ou composés. 
18 D'autres limites des classifications hiérarchiques ont été constatées, bien que celles-ci constituent pour Kwasnik (1999) d'excellentes représentations du savoir pour des domaines matures dans lesquels la nature des entités et la nature des relations importantes sont connues. Ces limites seront abordées dans la partie 3.3. de cet article.

19 Nous soutenons que la classification à facettes peut permettre de résoudre les problématiques liées à l'appartenance potentielle d'un objet à plusieurs classes, et celles qui sont liées à l'évolution rapide de l'environnement dans lequel les connaissances sont produites dans les entreprises.

\subsection{Classification génériques et spécifiques}

Les objectifs d'un système de classification sont aussi des facteurs déterminants pour la construction du système. Pour Beghtol (2001), toute classification est créée pour remplir un objectif, il n'y a pas de classification neutre vis-à-vis d'un but. Les systèmes de classification reflètent un point de vue, les assomptions qui gouvernent chaque système donnent le contexte général du système et jouent un rôle pour sa fondation sémantique. Pour Hjørland (2007), le critère pour la classification devrait être basé sur une compréhension des buts spécifiques, valeurs et intérêts en jeu. La construction d'une classification doit suivre ses buts, et différents objets peuvent être classés de différentes manières pour des buts différents, requérant des structures différentes (Denton, 2003).

21 Enfin Beghtol (2001) affirme que la complexité du monde du savoir ne permet plus de considérer un domaine comme stable ou unifié, les structures du savoir ayant changé avec la globalisation grandissante des échanges d'information. Il est donc nécessaire de repenser tous les aspects des systèmes de classification. Nous nous intéresserons particulièrement à l'aspect contextuel ou spécifique des classifications. En effet, le Classification Research Group (CRG), créé en 1952, a produit une littérature fournie sur les classifications à facettes et notamment sur des classifications spécifiques. Nous entendons par ce dernier terme, des systèmes de classification dont l'objectif n'est pas d'englober tout l'univers du savoir, comme les classifications des bibliothèques tentent généralement de le réaliser.

22 Ainsi, le CRG préfère observer une collection de documents et construire une classification appropriée à cette collection (Vickery, 1966). Vickery (1960) s'oppose à l'application mécanique et contrainte à un sujet de catégories fondamentales, qui doivent plutôt servir de guide pour suggérer des caractéristiques possibles qui ne devraient pas être ignorées (La Barre, 2010).

\subsection{Définitions et principes de la classification à facettes}

Créée en 1924 par S.R. Ranganathan, bibliothécaire et mathématicien indien, la classification à facettes facilite l'expression des sujets composés, ce que permettent difficilement les classifications traditionnelles énumératives, qui n'autorisent pas facilement la combinaison de termes provenant de différentes parties d'un schéma de classification (Spiteri, 1998). Dans ce nouveau modèle de classification, «les sujets des documents ne sont plus exprimés par un indice global tiré d'une liste existant à priori mais par une combinaison personnalisée d'indices-concepts » (Maniez, 1999, p. 250). Dans sa théorie de l'analyse facettes, publiée en 1933 et actualisée en 1967, Ranganathan a proposé des jeux de critères, de postulats et de principes pour la construction de schémas 
de classification à facettes, qu'il a appliqués dans la Colon Classification (CC). Celle-ci repose sur cinq facettes qui constituent le modèle PMEST (Personnalité, Matière, Energie, Espace, Temps), censé pouvoir décrire n'importe quel sujet, bien que Ranganathan reconnaissait qu'il n'y a pas de classification idéale ou universelle (Hudon et Mustafa El Hadi, 2010).

La notion de facette apparait souvent comme « l'apport théorique le plus important de ce siècle en science de l'information» (Maniez, 1999, p. 249). La classification à facettes est créditée de nombreux avantages dans la littérature. Ceux qui reviennent le plus souvent sont l'expressivité, la souplesse, la simplicité, la cohérence et l'adaptabilité (Ali Du, 2004 ; Maniez, 1999; Marleau et al., 2009). La classification à facettes est aussi reconnue comme favorisant le balayage (browsing), la navigation et la recherche d'information (Broughton, 2005). Broughton explique notamment que la classification à facettes permet le balayage (qui consiste à parcourir rapidement un corpus à l'aide, éventuellement, d'un SOC, pour savoir ce qu'il contient) grâce à sa structure logique et à sa capacité d'exprimer synthétiquement des sujets complexes ou composés. Sa structure compatible avec une interface utilisateur, et le fait qu'elle offre des points d'accès multiples vers les documents, favorisent la navigation à l'intérieur d'un corpus. Enfin la recherche est facilitée par le filtrage progressif basé sur des critères multiples (les facettes) (Broughton, 2006).

Pour Denton, la classification à facettes est «un jeu de catégories mutuellement exclusives et conjointement exhaustives, chacune construite en isolant une perspective sur les items (une facette) qui se combinent pour décrire entièrement tous les objets en question, et que l'utilisateur peut utiliser en cherchant ou en parcourant pour trouver ce dont il a besoin » (2003, p. 1).

Pour Beghtol (2008), la classification à facettes correspond à une approche analyticosynthétique qui s'apparente à un dispositif syntaxique et non sémantique. Pour Kwasnik (1999), elle repose sur la croyance qu'il y a plus qu'une seule manière de voir le monde et que même les classifications qui sont vues comme stables se révèlent provisoires et dynamiques. La classification à facettes est obtenue par le biais de l'analyse par facettes, une technique détaillée notamment dans La Barre (2010), Spiteri (1998) et Denton (2003). Globalement, il s'agit d'une technique en deux étapes ; la première correspond à l'analyse, qui consiste à déconstruire un sujet en ses diverses composantes, et la seconde, la synthèse, qui concerne la génération d'un indice significatif intégrant les différents composants du sujet en fonction de règles syntaxiques (Hudon et Mustafa El Hadi, 2010).

Une facette peut être définie comme l'une des dimensions d'analyse d'un sujet dans une classification multi-dimensionnelle, mais il est difficile pour les auteurs de s'accorder sur une définition, comme le montre Maniez (1999). Nous proposons des éléments de définition en partie 3.3.3 de cet article.

La classification à facettes fait usage du principe de coordination des concepts, qui permet de présenter le sujet d'un texte par la combinaison ou la synthèse de deux symboles ou plus, chacun représentant un concept indépendant. L'apport de la classification à facettes se situe au niveau du tri sur les regroupements de termes (Vickery, 1966).

Nous allons dans la partie suivante nous intéresser à l'adaptation des principes de la classification à facettes pour l'organisation des connaissances en entreprise. 


\section{Apports de notre approche de la classification à facettes pour l'organisation des connaissances en entreprises}

\subsection{Présentation et fonctionnement de l'outil}

Notre approche de la classification à facettes est largement contextuelle. Elle vise l'adaptation de principes de création de classification à facettes à chaque entreprise et n'a pas de visée universelle. L'outil en cours de développement permet de taguer des documents au fil de l'eau, lors de leur création ou ultérieurement, en rassemblant les tags dans une structure à facettes.

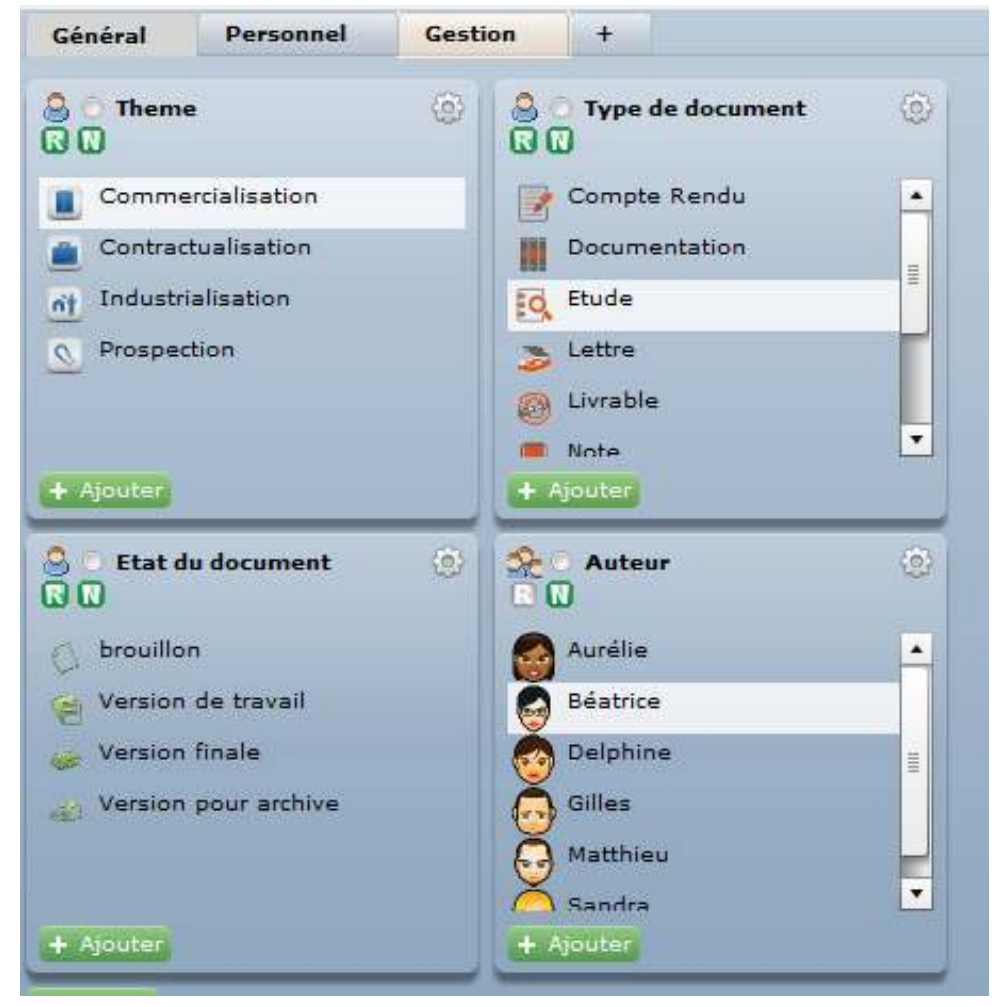

Figure 1 : Présentation de l'interface du SOC à facettes

Dans la figure 1, on peut observer quatre facettes ayant des valeurs correspondant au type d'information annoncé dans le titre de la facette (Thème, Type de document, État du document, Auteur). La sélection de valeurs de facettes (en surbrillance), que nous considérons comme des tags puisqu'elles sont créées à la volée et en langage naturel, par opposition aux langages contrôlés et aux termes imposés dans les classifications bibliographiques, permet d'ajouter ces tags comme métadonnées associées au document lors de son enregistrement. Ce tagging peut également contribuer ou non (il s'agit de fonctions paramétrables) au nommage du document et à son classement dans un dossier. Lors de l'assignation d'un ou plusieurs tags à une ressource documentaire, le document peut être classé dans un dossier choisi manuellement ou automatiquement. Dans ce dernier cas, le dossier est suggéré par le système, les recommandations étant basées sur un apprentissage automatique des corrélations entre des combinaisons de tags et des 
dossiers de classement. En haut de la figure, on observe les onglets Général, Personnel, Gestion : il s'agit de contextes qui rassemblent des jeux de facettes utilisées fréquemment ensemble. Ces contextes peuvent être construits en fonction d'activités mais aussi, éventuellement, d'autres éléments structurants (voir partie 3.2.3 de cet article).

On constate que notre système rassemble et intègre dans une seule et même opération les actions habituellement distinctes de nommer, indexer et classer un document. Lors de la recherche de documents, les facettes et les tags utilisés pour l'indexation - nommage classement sont présentés de manière identique dans l'interface de recherche. Quand l'utilisateur sélectionne un ou plusieurs tags, le système agit en filtrant progressivement les documents présents dans la base et ne donne comme résultats que les documents préalablement taggués par l'utilisateur pendant sa session de recherche. Par ailleurs, à chaque fois qu'un manque apparait dans la classification, l'utilisateur peut la réorganiser, c'est-à-dire repenser l'organisation de sa classification, de ses documents et de ses connaissances par l'ajout à la volée ou le retrait d'un contexte, d'une facette ou d'une valeur de facette.

\subsection{Une méthodologie ascendante de création de classification à facettes}

33 La méthodologie associée à l'outil est dite ascendante car elle permet une construction progressive du schéma de classification par les utilisateurs eux-mêmes, dans lequel certaines dimensions de description peuvent être partagées par un groupe métier, projet, ou au sein de l'ensemble de l'entreprise. Cela vise à rendre possible la récupération du savoir disséminé dans les différents lieux où celui-ci est consigné, comme il a été évoqué plus haut. Cette méthodologie s'inspire des méthodologies existantes de création de classification à facettes, présentées notamment par Spiteri (1998) et Denton (2003), bien qu'il n'y ait pas de système de notation, ni d'ordre de citation des facettes ou des valeurs de facettes. Les combinaisons de valeurs de facettes peuvent être spécifiques à chaque document.

Lors de l'utilisation de la classification à facettes évolutive et progressivement construite, l'acteur est en mesure de faire varier le degré de précision de sa documentarisation en fonction de ses objectifs. Dans la figure 2, le but du contexte présenté est la capitalisation des connaissances et on se trouve donc dans une démarche de gestion de connaissances ( Knowledge Management). Dans ce cas d'usage, l'utilisateur peut vouloir indexer un document, ici une étude sur la thématique du multimédia, sous forme de synthèse, dans le cadre d'un projet nommé Jarry, et vouloir se souvenir que la valeur ajoutée spécifique de ce document réside dans sa bibliographie. Avec notre outil, cet utilisateur pourra lui associer les tags suivants :

- Facette Type de document : Étude

- Facette Type de contenu : Synthèse

- Facette Thématique : Multimédia

- Facette Projet : Jarry

- Facette Intérêt du document : Bibliographie 
Figure 2 : Présentation d'une maquette pour un cas d'utilisation dans un objectif de KM

\section{Etudes \& Projets VEILLE KM SUPPORT}

\begin{tabular}{|c|c|c|c|}
\hline Type de document & Thénatique & Type de contenu & Intérêt du documen \\
\hline Etude & Multimédia & Synthèse & Exhaustif \\
\hline Proposition Connerciale & Ecologie & Cartographie & Conséquences \\
\hline Note & Urbanisation & Analyse & Réflexions \\
\hline Rapport & Démographie & Description & Perspectives \\
\hline$C R$ & TIC & Données d'entrée & Exemple \\
\hline Présentation & Econonie Energie & Chiffres & Conclusion \\
\hline Contrat & & Schéma & Remarques \\
\hline Lettre & & Modélisation & Retours \\
\hline \multirow[t]{2}{*}{ Livrable } & & Contribution & Bibliographie \\
\hline & & Liste & \\
\hline Etat du document & Projet & \multirow{2}{*}{$\begin{array}{l}\text { Export } \\
\text { Actualité }\end{array}$} & \\
\hline Document de travail & Jarry & & \\
\hline Version Intermédiaire & Natural & Diffusion (MV) & Confidentialité \\
\hline Version Finale & Ami & Interne & $C O$ \\
\hline \multirow[t]{4}{*}{ A Archiver } & Cassis & Collaboratif & $c 1$ \\
\hline & Koat & Partenaires & C2 \\
\hline & & Client & $C 3$ \\
\hline & & Site Web & $C 4$ \\
\hline
\end{tabular}

La difficulté de la méthodologie ascendante pour notre outil provient de plusieurs facteurs. Les méthodologies proposées par Vickery (1960), Spiteri (1998) et Denton (2003) sont considérées comme évaluatives plutôt que génératives par Wild et al. (2009). D'autres auteurs (Hudon et Mustafa El Hadi, 2010) pointent également le manque de guide méthodologique pour identifier les facettes pertinentes. D'une part, lorsqu'on cherche à appliquer les principes de la classification à facettes aux entreprises, on se heurte à des éléments hétérogènes à plusieurs niveaux: les services de l'entreprise n'ont pas nécessairement les mêmes besoins en termes de types d'information relatifs aux documents, selon qu'il s'agit de services support (ressources humaines, comptabilité) ou de services opérationnels, et ils ne traitent pas les mêmes types de documents. Il s'agit ici de préoccupations relatives aux activités métier. Les types de documents varient très fortement aussi, comme nous le verrons dans la partie suivante de cet article, et ces types de documents n'ont pas nécessairement besoin d'être documentarisés avec les mêmes informations. D'autre part, comme cela a été évoqué dans la partie 1, l'environnement de l'entreprise évolue rapidement et les tâches effectuées dans une activité métier sont renégociées, et subissent des modifications au cours du temps. La méthodologie vise à permettre aux utilisateurs de créer leur classification personnelle dans une démarche ascendante, pour favoriser l'émergence d'éléments utiles; elle n'a pas pour objectif l'imposition d'un schéma créé en amont à partir de catégories déterminées à priori, en fonction soit de schémas de classification existants soit du savoir déjà possédé dans le domaine, et qui correspond à une approche top-down. Enfin, les éléments sélectionnés pour la documentarisation, qui correspondent aux facettes et à leurs valeurs, peuvent servir à décrire des éléments contextuels relatifs à la situation de production des documents, mais aussi des caractéristiques abstraites (thématique ou sujet intérêt du document) plutôt que de se limiter à des attributs standards (date, auteur par exemple). 


\subsubsection{Importance de la notion de contexte}

Duant aux conditions de production du document à indexer ou, lors de la recherche, aux quant aux conditions de production du document à indexer ou, lors de la recherche, aux éléments présents dans la mémoire de l'utilisateur sur le document qu'il recherche et dont l'outil représente les traces correspondantes. Ainsi, nous pouvons dire que notre outil s'adapte au fonctionnement cognitif des utilisateurs, puisque tous ne mémorisent pas le même type d'informations à propos de leu r production documentaire (Pikas, 2007). Au cours d'une des études réalisées pour le projet, nous avons constaté que des ingénieurs chercheurs d'une firme industrielle mettent tous en place une organisation personnelle de leurs arborescences de fichiers et qu'ils rencontrent des difficultés à retrouver certains documents dans leur propre organisation des connaissances. Ces systèmes personnels de codification ou d'organisation des connaissances, s'ils ne sont pas soutenus par une application qui les encadre et les rend lisibles en leur offrant un environnement qui les contextualise, peuvent s'avérer difficilement exploitables pour d'autres utilisateurs, voire compromettre l'accès par les agents eux-mêmes à leurs propres ressources documentaires.

Par ailleurs, en poursuivant le parallèle entre les bibliothèques et les entreprises, plusieurs divergences sont à observer sur 1 a nature des corpus. Dans les premières, la collection est généralement homogène, le contenu est mature et le format pérenne puisque c'est le cycle de publication qui guide la production des documents. Dans les entreprises, les types de documents sont plus hétérogènes, leur production est liée, dans le cas de notre étude sur les ingénieurs chercheurs, aux activités métiers et au cycle temporel associé au processus d'étude, conception, production, réalisation. Dans cette étude, nous avons relevé plus de 110 types de documents différents et avec des occurrences très variables (Desfriches Doria, 2011), ce qui va dans le sens de ce qui est présenté par Wild et al. (2009), lesquels recensent plus de 250 genres de documents avec des occurrences de un à des milliers, dans leur étude d'une division d'ingénierie d'une entreprise de 60 salariés. Ces auteurs insistent sur l'importance de la documentation informelle dans la masse de la documentation globale et qui sert à documenter l'activité d'ingénierie, à communiquer, et aussi de moyen pour mener à bien les missions ainsi que pour la production de la documentation formelle (livrables). L'exploitation de cette

Études de communication, 39 | 2012 
documentation informelle (notes, correspondances...) nécessite un savoir tacite et une connaissance du contexte de production des documents. Ces observations nous incitent à penser que le partage des documents entre acteurs du même métier est de prime importance pour la capitalisation des connaissances dans l'entreprise.

\subsubsection{Le contexte : un élément dynamique} comprendre, contextualiser et désambigüiser des formes d'activités et d'informations, alors que sa nature est d'être continuellement négociée et redéfinie, d'où la nécessité de pouvoir réadapter le schéma de classification à tout moment, dans le contexte évolutif de l'entreprise.

41 Pour Kwasnik (1989), les classifications sont plutôt centrées sur la personne et la situation que sur les objets. Henderson (2005), dans son étude des noms de dossiers et de leur structure, dégage quatre dimensions prépondérantes pour décrire les documents dans le cadre de la gestion personnelle des documents (Personal Information Management) : il s'agit du genre de document, des tâches, du sujet et de l'aspect temporel.

Barreau (1995) note que le contexte dans lequel un document est acquis ou créé a beaucoup à voir avec la manière de le classer, de le stocker et de le retrouver ensuite. Le contexte, pour elle, comprend la nature de la tâche, le sujet, les outils disponibles, le public visé par le travail, l'usage visé de l'information. Elle reconnait par ailleurs la nature dynamique de l'environnement individuel, dans lequel les priorités changent et modifient la nature du travail, ainsi que l'utilité des systèmes de gestion de documents multidimensionnels.

Pour Wild et al. (2009), les documents sont embarqués dans un contexte et contiennent des représentations des aspects tangibles et intangibles du monde. Dans sa tentative de développement d'une classification à facettes pour la documentation d'ingénierie, l'auteur remarque des facettes décrivant des aspects plus situationnels (contextuels), comme les manifestations, le statut de partage, les mécanismes de manifestation et de partage, et d'autres plus interprétatifs comme le genre et le but.

Pour Dourish (2004), le monde tel que nous le percevons est essentiellement un consensus sur son interprétation, les catégories abstraites sont des éléments qui s'imposent au monde par le biais de nos interactions avec celui-ci et avec chacun, plutôt que des éléments qui existent à l'intérieur du monde. Il voit donc la notion de contexte comme un problème d'interaction. $\mathrm{P}$ our lui, la contextualité est une propriété relationnelle qui se tient entre les objets et les activités et elle est définie dynamiquement. Le contexte est particulier à chaque occasion d'activité et d'action. C'est une propriété occasionnelle, pertinente en fonction de paramètres particuliers, de cas particuliers d'actions et de parties particulières de cette action. Enfin, le contexte résulte de l'activité, il est activement produit, maintenu, et ordonné dans le cours de l'activité. Pour Dourish, la détermination de la contextualité ne peut être faite à priori. C'est une caractéristique émergente de l'interaction, déterminée dans le moment et dans l'action (en la réalisant).

\subsection{3 Éléments de contexte structurant la classification à facettes}

Nous accordons toute son importance à la notion de contexte et elle trouve son application fonctionnelle dans l'outil à travers l'usage des onglets. Ceux-ci peuvent être 
créés en fonction d'activités, comme dans la Figure 3, ou d'autres éléments structurant les pratiques métiers des utilisateurs comme :

Öle type de collectif partageant des documents: entreprise, métier, projet, individuel ou informel;

Öles besoins: variables à ajuster en fonction d'aspects contingents, comme la commodité, l'urgence ;

Ö les usages : en fonction des choix et habitudes des utilisateurs;

Ö le corpus : en fonction des types de documents à indexer;

Öla structuration des activités: une organisation pouvant opérer dans plusieurs domaines d'exercices;

Öla structure organisationnelle globale: en fonction des grands services dans l'organisation;

Ö les phases d'un projet : découpage en lots ou en grandes phases.

Ces éléments peuvent être combinés et avec des niveaux de granularité variables pour la création de contextes pertinents en termes d'usages.

La figure 3 présente une partie de l'interface de notre outil, configuré pour une activité spécifique : la veille.

Figure 3 : Exemple de Classification à facettes pour la veille

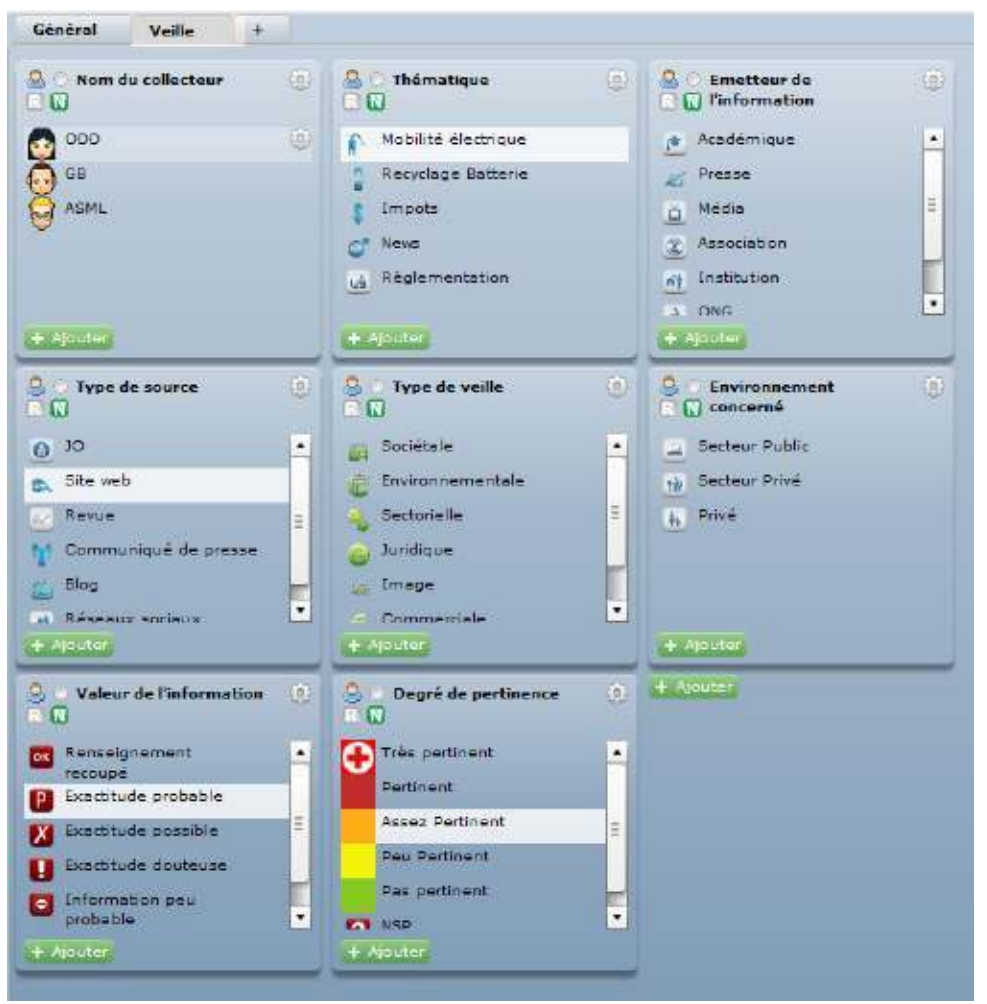

48 Nous considérons par ailleurs qu'un contexte peut s'apparenter à un point de vue. En effet, l'ensemble des facettes rassemblées dans un contexte reflète une activité ou un usage particulier, et l'on s'écarte ainsi de l'indexation standardisée pratiquée dans les bibliothèques où les notices comportent toujours les mêmes types d'information. L'outil que nous développons est adaptable à n'importe quel besoin en termes de types d'information, ce qu'il propose étant une structure syntaxique multidimensionnelle. L'utilisateur est libre de composer les libellés des contextes, facettes et tags ainsi que de déterminer les éléments pertinents pour la structuration de la classification à facettes. La 
méthodologie de création de classification à facettes vise à guider l'utilisateur dans l'élaboration de son s chéma de classification à facettes, basé sur les contextes, les facettes et les tags (valeurs de facettes).

\subsection{Synthèse des apports, limites et difficultés des classifications à facettes}

Dans cette partie nous présentons les avantages de la classification à facettes implémentée dans le SOC sur lequel nous travaillons, avant d'expliquer comment les principes de la classification à facettes ainsi que notre outil peuvent apporter des réponses aux limites des classifications bibliographiques. Nous abordons enfin les principales difficultés résiduelles.

\subsubsection{Avantages de la classification à facettes en référence à notre outil}

En reprenant les avantages les plus cités de la classification à facettes, il est possible de les détailler en prenant comme référence l'outil que nous développons.

L'expressivité : cette qualité est liée à la combinaison des tags qui permet d'exprimer des sujets ou concepts complexes, au fait de pouvoir créer les libellés des tags librement, ainsi qu'à la possibilité d'assigner autant de tags que l'utilisateur le juge nécessaire.

La souplesse : cette qualité est due à la possibilité d'ajout et de suppression de contextes, facettes et tags à la volée.

53 La simplicité: l'ergonomie de l'outil ainsi que le fonctionnement syntaxique, qui peut accueillir tous types de valeurs pour les facettes, selon les besoins, en font un outil facile à utiliser.

54 La cohérence : le fonctionnement du système est régulier, la structure transverse est toujours basée sur le même principe de complémentarité des dimensions de description, et leur combinaison produit un jeu de métadonnées complètes et adaptables en fonction des besoins.

L'adaptabilité: la structure peut être paramétrée à tous les niveaux, les choix effectués concernant les éléments structurants sont faits en fonction des besoins des utilisateurs individuels, des groupes ou organisations entières.

\subsubsection{Apports des classifications à facettes face aux limites des classifications bibliographiques}

Le tableau 1 présente les réponses apportées par les principes de la classification à facettes, ainsi que par notre outil, aux problèmes classiques des classifications bibliographiques. Les limites de ces dernières sont exposées dans la première colonne du tableau. 
Tableau 1 : Synthèse des apports de la classification à facettes par rapport aux limites des classifications bibliographiques

\begin{tabular}{|c|c|c|}
\hline $\begin{array}{c}\text { Limites des Classifications } \\
\text { bibliographiques }\end{array}$ & $\begin{array}{c}\text { Solutions potentielles proposées dans } \\
\text { les principes des classifications à } \\
\text { facettes (d'après (Kwasnik, 1999) et } \\
\text { (Hudon \& El Hadi, 2010) }\end{array}$ & $\begin{array}{l}\text { Solutions fonctionnelles apportees par la } \\
\text { classification à facettes dans notre SOC }\end{array}$ \\
\hline $\begin{array}{l}\text { Elles proposent un point de vue unique sur } \\
\text { les objets à classer }\end{array}$ & $\begin{array}{l}\text { Les liens sont ponctuels, fonctionnels et } \\
\text { pragmariques entre concepts ou sujets } \\
\text { (possibilité de varier les perspectives ou } \\
\text { points de vue) }\end{array}$ & $\begin{array}{l}\text { Un jeu de facettes peut representer un point } \\
\text { de vue, dans notre systeme lutilisateur peut } \\
\text { disposer de plusieurs jeux de facettes } \\
\text { distincts en fonction de ses activites ou } \\
\text { d'autres elements contextuels importants }\end{array}$ \\
\hline Un seul critère de division y est toléré & \begin{tabular}{|c|}
$\begin{array}{c}\text { Le principe de la classification à facettes } \\
\text { est d'appliquer plusieurs critères de division } \\
\text { simultanément }\end{array}$ \\
\end{tabular} & $\begin{array}{c}\text { Le principe de la classification à facettes est } \\
\text { d'appliquer plusieurs critères de division } \\
\text { simultanciment }\end{array}$ \\
\hline $\begin{array}{l}\text { Pour les élaborer, le créateur de la } \\
\text { classification a besoin de disposer diun } \\
\text { savoir global et complet sur le domaine }\end{array}$ & $\begin{array}{c}\text { Ele ne nécessite pas ui savoir complet sur } \\
\text { le domaine, ce qui est utile pour des champs } \\
\text { emergents ou qui changent }\end{array}$ & $\begin{array}{c}\text { Possibilité d'alimenter progressivement la } \\
\text { classification }\end{array}$ \\
\hline $\begin{array}{c}\text { Toutes les entités d'une classe dóvent } \\
\text { présenter le meme niveau conceptuel de } \\
\text { granularité }\end{array}$ & $\begin{array}{c}\text { En principe la classification à facette } \\
\text { applique aussi cetre règle }\end{array}$ & $\begin{array}{l}\text { En fonction du type dinformation represente } \\
\text { dans une facette, des besoins de lutilisateur, } \\
\text { et des contr aintes, la granularite peut varier }\end{array}$ \\
\hline $\begin{array}{l}\text { La transitivité peut devenir une contrainte } \\
\text { (comme les attributs d'une classe sont } \\
\text { hétités, les sous classes sont des membres } \\
\text { de la classe supérieure mais aussi de toutes } \\
\text { les classes supérieures) }\end{array}$ & $\begin{array}{l}\text { Les composantes restent indépendantes } \\
\text { dans une classification à facettes }\end{array}$ & $\begin{array}{c}\text { Pas de notion d'héritage dans la classification } \\
\text { a facettes }\end{array}$ \\
\hline $\begin{array}{c}\text { L'exclusivité mutuelle : une entité ne peut } \\
\text { appartenir qu'à une seule classe }\end{array}$ & $\begin{array}{c}\text { Appartenance possible d'un objet a plusieurs } \\
\text { classes, la complementarite des categocies } \\
\text { etant un principe fondamental de la } \\
\text { classification à facettes }\end{array}$ & $\begin{array}{c}\text { Appartenance possible d'un objet à plusieurs } \\
\text { classes, la complementarite des categories } \\
\text { etant un principe fondamental de la } \\
\text { classification a facettes }\end{array}$ \\
\hline $\begin{array}{l}\text { Les règles d inchusion dans les classes } \\
\text { peuvent s'averer trop rigides }\end{array}$ & $\begin{array}{l}\text { La structure classificatoire est lâche ct } \\
\text { extensible }\end{array}$ & $\begin{array}{l}\text { I inclusion dans une classe est moms rigide } \\
\text { et le systeme permet d'associer deux valeurs } \\
\text { d'une meme facette à une ressource }\end{array}$ \\
\hline
\end{tabular}

\subsubsection{Difficultés liées aux classifications à facettes et apports théoriques}

Notre SOC tolère donc des éléments hétérogènes pour les valeurs d'une même facette tant
que cela reste cohérent pour l'utilisateur. Ainsi, il se rapproche des outils de tagging de
ressources en ligne, comme les outils d'indexation collaborative, qui dans l'espace vide
que constitue un tag, voient venir s'inscrire des labels de types variables, tant au niveau
des usages que des types d'entités mentionnées. La variabilité des tags dans notre SOC
peut se caractériser, de manière non exhaustive, comme suit:

Les principales difficultés liées à l'élaboration d'une classification à facettes sont énumérées dans cette partie et nous tentons d'y apporter des solutions en référence au fonctionnement de notre outil. Ces difficultés sont abordées dans Hudon et Mustafa El Hadi, (2010) et Wild et al. (2009).

La première difficulté consiste à établir des facettes appropriées; elle est liée aux ambiguïtés sur la nature et la fonction des facettes, qui aboutissent à des interprétations divergentes de la notion même. Selon nous, la facette peut se définir comme une catégorie qui décrit une propriété des documents parmi d'autres propriétés complémentaires. Une facette rassemble des tags qui traitent le même type d'information relativement à un contexte (activité ou autre), le type d'information dans une facette est homogène, mais la granularité ou le degré de spécificité des valeurs ainsi que leurs libellés peuvent varier. La création d'une facette relève d'une forme de régularité au sein du corpus : on crée une facette quand plusieurs éléments du corpus ont des propriétés en commun qu'on estime importantes et en fonction des besoins de description de la situation de production des documents. 
Ö variation grammaticale : noms, verbes, adjectifs ;

Ö variation au niveau du type d'entité: une facette «Colloques» pourrait avoir pour valeurs des noms de lieux, des noms de colloques, des noms de personnes organisatrices de colloques ;

Ö variation sémantique : les mêmes noms de villes pourraient être utilisés dans deux facettes différentes pour désigner, dans un cas, des informations relatives à des colloques et dans l'autre, des informations de type purement géographique ;

Ö variation du niveau de granularité: une facette géographique, par exemple, pourrait avoir pour valeurs des noms de pays, de villes, de rues.

60 Selon nous, la fonction des facettes est de servir à la représentation du contenu d'un document un document et à l'ajout de métadonnées descriptives, éventuellement de contribuer à le nommer et à le classer. La facette sert aussi à décrire la situation de production ou d'utilisation du document, et son contenu en fonction des objectifs de la classification. Elle permet une visualisation des éléments d'indexation et une vue d'ensemble des catégories et de leurs valeurs en fonction des contextes (onglets).

Chaque facette est donc vue comme une classe non exclusive (il est possible d'attribuer deux valeurs provenant d'une même facette) par le biais de laquelle on ajoute des métadonnées et on classe un document via une ou plusieurs de ses propriétés. Le document a généralement plusieurs propriétés et peut être indexé à l'aide de plusieurs c lasses à la fois. Les valeurs de facettes (les tags) sont rassemblées parce qu'elles servent à décrire une propriété commune d'éléments du corpus considéré. Le type d'information décrit par une facette, dont les valeurs (les tags) sont des occurrences, est identifié à l'aide du titre de la facette.

62 L'exclusivité mutuelle classique dans les systèmes de classification à facettes traditionnels, se situe dans notre approche, au niveau du type d'information traité par la facette, ce qui implique que le même type d'information sera toujours traité par la même facette.

63 Le deuxième problème soulevé par Hudon et Mustafa El Hadi (2010) est le manque de relation entre facettes. Nous considérons que cet aspect n'est pas nécessaire et fait perdre les qualités d'autonomie et d'indépendance des facettes, qui permettent des croisement $\mathrm{s}$ inédits de dimensions d'indexation et de critères de recherche. Des systèmes de classification à facettes intégrant des relations entre facettes existent (Marleau et al., 2009), comme le modèle ISIS, mais nous considérons qu'il bride les possibilités de combinaison de valeurs de facettes. De plus, ce modèle formel ne laisse pas l'utilisateur libre de créer son schéma de classification, puisque celui-ci prend effet dans une démarche top-down, qui consiste en une analyse précise de la structure organisationnelle de l'entreprise ainsi que des processus d'affaires (business process) modélisés.

La troisième difficulté correspond à des problèmes de visualisation du schéma global (Hudon et Mustafa El Hadi, 2010). Grâce aux contextes (onglets), qui rassemblent les facettes en fonction d'un principe d'organisation et de structuration logique pour l'utilisateur et son activité telle qu'il l'exerce, ce dernier est en mesure de visualiser toutes les facettes dont il a besoin au moment de l'indexation ou de la recherche de documents, si le schéma est bien élaboré.

Un autre problème relevé par les mêmes auteurs est le risque d'une vision trop locale. Il s'agit du principal problème auquel la méthodologie sur laquelle nous travaillons doit pouvoir apporter des réponses. Pour choisir un degré approprié de précision ou de profondeur de l'indexation, la tentation est présente de trop détailler les valeurs de facettes et de multiplier celles-ci. La recommandation principale consiste à tenter de 
garder la cohérence de la classification comme objectif lors de sa maintenance. Pour ce faire, l'utilisateur doit préserver un niveau de spécificité homogène au moins au sein des contextes et ne pas focaliser sur un document mais l'insérer dans la dimension du corpus. Idéalement, lors de la création d'un tag l'utilisateur doit d'abord vérifier qu'il n'y a pas un autre terme équivalent ou qui se rapproche de cel ui qu'il veut créer. La question type qu'il devrait se poser lors de la création d'un tag est la suivante : «Si je crée ce tag, est ce qu'il va me resservir pour d'autres documents?». Si la réponse à cette question est négative, il faut alors tenter de trouver une formulation plus générique, car nous considérons qu'un cas d'utilisation unique d'un tag n'a pas sa place dans la classification à facettes. Le risque, si l'utilisateur ne respecte pas ce principe, est d'aboutir à une classification trop détaillée, trop précise et inutilisable sur un moyen terme par sa densité et son ampleur.

Un dernier problème abordé par Wild et al. (2009), est la question de la démarche de construction des facettes purement descendante ou ascendante (top down versus bottom up). Cette interrogation revient à se demander si l'on doit construire le schéma à partir des connaissances et catégories préexistantes sur un domaine (démarche descendante), ou à partir de l'étude d'un corpus de document (démarche ascendante). Nous considérons qu'il peut y avoir des formes de construction hybrides, étant donné qu'un utilisateur qui va élaborer une classification à facettes avec notre outil possède déjà une connaissance de son métier et de son milieu de travail, et qu'il réfléchit déjà avec des formes de catégorisation inhérentes à la réalisation de ses missions. Lorsque le schéma est créé par une personne extérieure à l'entreprise, nous préconisons une méthode d'entretien et d'observation des postes individuels de travail et des arborescences de fichiers existant s, complétée d'une éventuelle étude de corpus notamment pour la validation du schéma.

D'autres problématiques que nous ne pouvons traiter ici sont abordées par Wild et al. (2009) : la confusion entre l'analyse par facettes et la classification à facettes dans la littérature et le fait d'utiliser des domaines simples pour illustrer et expliquer les principes des classifications à facettes dans les méthodologies existantes, qui ne correspondent pas à la complexité réelle des situations d'utilisation des facettes, notamment parce qu'elles se concentrent sur la définition de facettes correspondant à des attributs standards, plutôt qu'à des caractéristiques abstraites. Enfin, l'entretien d'une illusion sur le fait que la reconnaissance de facettes pertinentes n'est pas problématique aboutit à un manque de directives méthodologiques sur l'élaboration des schémas de classification à facettes. Nous tentons dans notre travail méthodologique d'apporter des élément $s$ de clarification sur ces aspects.

\section{Conclusion}

La principale difficulté dans l'utilisation d'une classification à facettes adaptée au milieu de l'entreprise, telle que notre SOC le propose, réside dans le fait de passer d'un niveau global d'organisation des connaissances (le niveau du schéma de la classification) à un niveau plus détaillé pour le renseignement des métadonnées propres à un document ou à une situation d'activité de travail tout en veillant à ne pas générer trop de facettes ou de valeurs de facettes, afin de garder la cohérence globale du schéma de classification. Deux opérations différentes sont demandées à l'utilisateur, celles d'utiliser le système et d'en assurer la maintenance. Cette dernière activité correspond à une méta-activité. Enfin nous observons que le modèle PMEST proposé par Ranganathan présente un fort degré 
d'abstraction, du fait qu'il visait une certaine universalité. Plus un système vise l'universalité plus le degré d' abstraction augmente et nécessite une interprétation de la part des utilisateurs, ce qui peut éventuellement donner lieu à des erreurs ou approximations. En définitive, dans une classification à facettes, plus le degré de détail voulu est élevé pour décrire des sujets complexes, plus il est nécessaire d'ajouter des catégories. Nous pensons comme Vickery (1966), que chaque schéma de classification est spécifique, son application étant restreinte aux intérêts d'un groupe relativement homogène d'utilisateurs, qui pourrait correspondre dans le milieu de l'entreprise, à des communautés métiers. Ceci permettrait de réduire le degré d'abstraction du schéma de classification.

\section{BIBLIOGRAPHIE}

Ali F. M. et Du W., (2004), Toward reuse of object-oriented software design models, Information and Software Technology, vol. 46, n 8, pp. 499-517.

Argyris C., (1995), Savoir pour agir. Surmonter les obstacles à l'apprentissage organisationnel, Paris, InterÉditions ; Dunod.

Barreau D. K, (1995), Context as a Factor in Personal Information Management Systems, Journal of the American Society for Information Science, vol. 46, n 5, pp. 327-339.

Beghtol C., (2001), Relationships in classificatory structure and meaning, in Relationships in the organization of knowledge, (dir. Carol A. Bean et Rebecca Green), éd. Kluwer Academic Publishers.

Beghtol C., (2008), From the universe of knowledge to the universe of concepts : The structural revolution in classification for information retrieval, Axiomathes, vol. 18, pp. 131-144.

Boydens I., (2010), Hiérarchie et anarchie, Dépasser l'opposition entre organisation centralisée et distribuée ?, Les cahiers du numérique, vol. 6, n³ 3p. 77-101.

Broughton V., (2006), The need for a faceted classification as the basis of all methods of information retrieval, Aslib Proceedings, New Information Perspectives, vol. 58, n 1-2, pp. 49-72.

Cotte D., (2007), L'organisation des connaissances entre le formalisme des outils et la complexité des représentations : une illustration par le cas des portails d'entreprise, Sixième Congrès du chapitre français de l'ISKO, Organisation des connaissances et société des savoirs : concepts, usages, acteurs, 7-8 juin 2007, Toulouse, http://www.isko-france.asso.fr/pdf/isko2007/Actes\%20ISKO\% 20FR\%202007\%20p\%2063-78.pdf, visité le 24 avril 2012.

Denton W., (2003), How to make classification and put it on the web, http://www.miskatonic.org/ library/facet-web-howto.html, visité le 24 avril 2012.

Desfriches Doria O., (2011), Systèmes d'Organisation des Connaissances hétérogènes et pratiques de gestion de l'information, Actes du $8^{\mathrm{e}}$ Colloque International de l'ISKO France, Stabilité et dynamisme dans l'organisation des connaissances, 27-28 Juin 2011, Lille, Université Charles-deGaulle - Lille 3. 
Dourish P., (2004), What we talk about when we talk about context, Personal and Ubiquitous Computing, vol. 8, n 1, 19-30, http://www.csc.kth.se/utbildning/kth/kurser/DH2622/mdifk11/ inlamningsuppgifter/Dourish.pdf, visité le 24 avril 2012.

Ermine J.-L., (2000), Les systèmes de connaissances, Paris, Hermès Science publications.

Gandon F. et Dieng-Kuntz R., (2005), Chapitre 7 : Ontologie pour un système multi-agents dédié à une mémoire d'entreprise, Ingénierie des connaissances, Paris, L'Harmattan, pp. 141-163.

Grundstein M., (2002), De la capitalisation des connaissances au renforcement des compéntences dans l'entreprise étendue, $1^{\mathrm{er}}$ Colloque Gestion des Compétences et des Connaissances (GCC), « Vers l'articulation entre compétences et connaissances ", 12-13 décembre 2002, Nantes, France, http://basepub.dauphine.fr/handle/123456789/4143, visité le 24 avril 2012.

Grundstein M., (2003), De la capitalisation des connaissances au management des connaissances dans l'entreprise, Les fondamentaux du knowledge management, http://basepub.dauphine.fr/bitstream/ handle/123456789/2620/INTKM0304_finalversion_modifiee.pdf?sequence=2, visité le 24 avril 2012.

Henderson S., (2005), Genre, task, topic and time : facets of personal digital document management, in Proceedings of the $6^{\text {th }}$ ACM SIGCHI New Zealand chapter's international conference on Computerhuman interaction : making CHI natural (CHINZ ‘05), 2005, New York, NY, ACM, pp. 75-82.

Hjørland B., (2008), What is knowledge organization (KO) ?, Knowledge Organization, vol. 35, $\mathrm{n}^{\circ} \mathrm{2} / 3$, pp. 86-101.

Hjørland B., (2007), Semantics and knowledge organization, Annual review of Information Science and Technology, vol. 41, Issue 1, pp. 367-405.

Hudon M. et Mustafa El Hadi W., (2010), Organisation des connaissances et des ressources documentaires, De l'organisation hiérarchique centralisée à l'organisation sociale distribuée, Les Cahiers du Numérique, vol. 6, $\mathrm{n}^{\circ}$ 3, pp. 9-38.

Kwasnik B. H., (1989), How a personal document's intended use or purpose affects its classification in an office, in Proceedings of the $12^{\text {th }}$ annual international ACM SIGIR conference on Research and development in information retrieval (SIGIR ‘89), N. J. Belkin and C. J. van Rijsbergen (éds.), New York, NY, ACM, pp. 207-210.

Kwasnik B. H., (1999), The role of classification in knowledge representation and discovery, Library Trends, vol. 38, n 1, pp. 22-47.

La Barre K., (2010), A Semantic (faceted) Web ?, Les cahiers du numérique, vol. 6, nº 3, pp. 103-131.

La Barre K., (2012), Facet Analysis, Annual review of Information Science and Technology, vol. 44, Issue 1, pp. 243-284.

Mahé S., (2005), PUMEO : un modèle actif pour la gestion des connaissances tacites et explicites dans l'entreprise, Ingénierie des connaissances, (coord. R. Teulier, J. Charlet et P. Tchounikine), Paris, L'Harmattan, pp. 321-344.

Maniez J., (1999), Des classifications aux thésaurus, Du bon usage des facettes, Documentaliste Sciences de l'information, vol. 36, $\mathrm{n}^{\circ}$ 4-5, pp. 249-262.

Marleau Y., Mas S. et Zacklad M., (2008), Exploitation des facettes et des ontologies sémiotiques pour la gestion documentaire, in Broudoux Evelyne et Chartron Ghislaine (dirs.), Actes de la deuxième conférence Document numérique et Société, Traitements et pratiques documentaires : vers un changement de paradigme ?, 17-18 novembre 2008, Paris, France. Paris : ADBS (Collection Science et techniques de l'information), pp. 91-110. 
Mills J., (2004), Faceted classification and logical division in information retrieval, Library Trends, vol. 52, $\mathrm{n}^{\circ} 3$, pp. 541-570.

Nonaka I. et Takeushi H., (1997), La connaissance créatrice - La dynamique de l'entreprise apprenante, Bruxelles, De Boeck.

Pikas C. K., (2007), Personal information management strategies and tactics used by senior engineers, Proceedings of the American Society for Information Science and Technology, vol. 44, Issue 1, pp. 1-21.

Sauvagnac C., Falzon P. et Leblond R., (2000), La mémoire organisationnelle : reconstruction du passé, construction du futur, Ingénierie des connaissances, Évolutions récentes et nouveaux défis, (coord. Charlet Jean, Zacklad Manuel, Kassel Gilles et Bourigault Didier), Paris, Eyrolles, pp. 441-450.

Spiteri L., (1995), The Classification Research Group and the Theory of integrative levels, Katharine Sharp Review, Issue 1, http://mirrored.ukoln.ac.uk/lis-journals/review/review/summer1995/ spiteri.pdf, visité le 24 avril 2012.

Spiteri L., (1998), A simplified model for facet analysis, Canadian Journal of Information and Library Science, vol. 23, pp. 1-30.

Teulier R. et Girard N., (2005), Modéliser les connaissances pour l'action dans les organisations, Ingénierie des connaissances, (coord. R. Teulier, J. Charlet et P. Tchounikine), Paris, L'Harmattan, pp. 389-412.

Tort F., Teulier R., Grozs G. et Charlet J., (2005), Ingénierie des besoins, ingénierie des connaissances : complémentarité des approches de modélisation, Ingénierie des connaissances, (coord. R. Teulier, J. Charlet et P. Tchounikine), Paris, L'Harmattan, pp. 207-228.

Vickery B. C., (1960), Faceted classification, a guide to construction and use of special schemes, London, Aslib.

Vickery B. C., (1966), Faceted Classification schemes, New Brunswick, NJ, Rutgers University Press.

Vickery B. C., (2008a), The structure of subject classification for document retrieval, http:// www.iskoi.org/ilc////vickery.php, visité le 24 avril 2012.

Vickery B. C., (2008b), Faceted classification for the Web, Axiomathes, vol. 18, nº 2.

Wild P. J., Giess M. D. et McMahon C. A., (2009), Describing engineering documents with faceted approaches, Observations and reflections, Journal of Documentation, vol. 65, n 3, pp. 420-445.

Zacklad M., (2005), Processus de documentarisation dans les Documents pour l'Action (DopA) : statut des annotations et technologies de la coopération associées, Actes du colloque « Le numérique : Impact sur le cycle de vie du document pour une analyse interdisciplinaire », 13-15 Octobre 2004, Montréal, Québec, Villeurbanne, ENSSIB.

\section{RÉSUMÉS}

Cet article cherche à éclaircir dans quelle mesure la classification à facettes peut servir d'outil d'organisation des connaissances dans l'entreprise. Nous rappelons d'abord les besoins actuels des organisations en matière d'organisation des connaissances, puis nous focalisons sur l'approche traditionnelle de la classification à facettes; enfin, nous développons une approche adaptée de la classification à facettes pour l'organisation des connaissances dans l'entreprise, en présentant une classification à facette évolutive basée sur le tagging de documents. 
This paper suggests exploring the potential of faceted classification structures to serve as tools for knowledge organization in enterprises. We first examine current needs for knowledge organization in these enterprises. We then focus on traditional approaches to faceted classification. Finally, we develop a new approach to faceted classification adapted to the enterprise context, and propose a tool design that would enable the gradual development of the faceted structure based on the tagging of documents.

INDEX

Mots-clés : classification à facettes, organisation des connaissances, classification, classification bibliographique, classification spécifique, tagging

Keywords : faceted classification, knowledge organization, classification, bibliographic classification, specific classification, tagging

\section{AUTEUR}

\section{ORÉLIE DESFRICHES DORIA}

CNAM - Laboratoire DICEN

(Dispositif d'Information Communication à l'Ere du Numérique) 